Acetatbestimmung in verschiedenen Körperflüssigkeiten

Als Anwendungsbeispiel der Methode sind in Tabelle 3 die Ergebnisse von Acetatbestimmungen in Plasmaund Harnproben sowie im Liquor cerebrospinalis wiedergegeben. Vergleicht man die Mittelwerte, so ergibt sich ein recht großer Streubereich. Die Mittelwerte liegen bei $40,1 \mathrm{nMol} / \mathrm{m} l$ Plasma $(s= \pm 20,9), 25,0 \mathrm{nMol} /$ $\mathrm{m} l$ Harn $(\mathrm{s}= \pm 8,7)$ und $42,9 \mathrm{nMol} / \mathrm{m} l$ Liquor $(\mathrm{s}=$ 27,7). Bisher sind beim Menschen lediglich Plasmaacetatspiegel enzymatisch gemessen worden $(17,18)$. Sie liegen mit Werten $z$ wischen $50-100 \mathrm{nMol} / \mathrm{m} l$ etwas höher als die hier gaschromatographisch gefundenen. Im Vergleich zu den langkettigen unveresterten Plasmafettsäuren macht die freie Essigsäure demnach etwa den zehnten Teil aus. Vergleichbare Bestimmungen des Harn- und Liquoracetats liegen in der Literatur nicht vor.

Auf Grund unserer Erfahrungen können wir die hier beschriebene gaschromatographische Methode zur Acetatbestimmung gegenüber dem enzymatischen Sulfanil-
amid-Acetylierungstest sowohl im Hinblick auf die einfachere Handhabung als auch hinsichtlich der Reproduzierbarkeit der Ergebnisse als einen eindeutigen Fortschritt ansehen. Eine weitere Vereinfachung durch Verzicht auf die vorausgehende Abtrennung der Essigsäure durch Mikrodestillation, wie von anderer Seite beschrieben wurde $(4,14)$, erscheint uns nicht zweckmäßig. Bei direktem Einsatz von enteiweißten und eingeengten Proben werden nämlich im biologischen Material vorkommende höhere Homologen unter unseren Bedingungen mitaufgezeichnet, was bei den zum Teil erheblichen Retentionszeiten einen wesentlich größeren Zeitaufwand pro Analyse bedeuten würde. Die durch Auslassen der Mikrodestillation erreichte Zeitersparnis ginge damit wieder verloren. Hinzu kommt, daß der Injektor durch nicht flüchtige Bestandteile rasch verunreinigt wird.

Die Arbeit wurde mit Unterstützung der Deutschen Forschungsgemeinschaft Bad Godesberg (Schwerpunktprogramm „Ernährungsforschung ") durchgeführt. Für wertvolle methodische Hinweise danken wir Herrn Dr. B. Hampreckir und Herrn Dr. R. SeIfert am Max Planck Institut fụ̈r Zellechemie, Münçhen.

\title{
Literatur
}

1. Lundeuist, F., U. Fugmann und H. Rasmussen, Biochem. J., 80, 393 (1961). - 2. Conway, E. J., Mikrodiffusion Analysis, 4. Aufl., Crosby, Lockwood and Son I.td., London (1957). 3. Bergmeyer, H. U. und H. Moellering, Biochem. Z., 344, 167 (1966). - 4. MEdzIHRAdsky, F. und W. LAMPrecht, HoppeSeylers Z. physiol. Chem., 343, 35 (1965). - 5. BARtLEX, W., Biochem. J., 53, 305 (1953). - 6. Boetrger, I., U. Deutrcke, E. Prüsse-Evertz, B. D. Ross und O. Wreland, Zschr. exper. Med., 145, 346 (1968).-7.HochHeuser, H., H. WeIsS und O. WIELAND, diese Z. 6, 175 (1964). - 8. BAKER, R. A., J. Gas Chromatog., 4, 418 (1966). - 9. Appleby, A. J. und J. E. O. MAYNE, J. Gas Chromatog., 5, 211 (1967). - 10. Kaplanoví, B. und J.
JanÁx, Mikrochịm. Acta, 1-2, 119 (1966). - 11. Lough, A. K., R. S. Reid, M. Murray und F. M. Black, J. Sci. Food Agric., 18, 214 (1967). - 12. Kung, J. T., R. P. Naught und J. A. YERANSian, J. Food Sci., 32, 455 (1967). - 13. SallmanN, H. P. und K. H. Niesar, Z. Tierphysiol. Tierern., 23, 6 (1967). - 14. Baumgardr, B. R., Departmental Bulletin 1, Dptm. Dairy Science, Univ. of Wisconsin, Madison (1964). - 15. KArser, R., Chromatographie in der Gasphase, Bibliographisches Institut Mannheim (1962). - 16. Hokning, E. C., E. A. Moscatelit und C. C. Sweelex, Chem. and Ind., 1959, 751. - 17. LundQuist, F., Acta physiol. Scand., 50, 175 (1960). - 18. Werss, H., Dissertation München (1965).

\section{Fluorometrische Bestimmung von Testosteron im menschlichen Plasma}

\author{
Von V. Graef und HJ. Staudinger \\ Aus dem Physiologisch-Chemischen Institut der Universität Gießen (Direktor: Prof. Dr. Hj. Staudinger)
}

. (Eingegangen am 29. März 1968)

Herrn Prof. Dr. med. Dr. rer. nat. Ernst Schütte qum 60. Geburtstag gewidmet

Es wird eine Methode zur Bestimmung von Testosteron im Plasma des Menschen beschrieben. Bei dieser Methode wird Testosteron durch Dünnschichtchromatographie abgetrennt. Die Fluoreszenz-Reaktion auf einer Oberfläche von Lithiumhydiroxid dient zur quantitativen Bestimmung. Durch diese Methode kann noch 0,01 $\mu \mathrm{g}$ Testosteron bestimmt werden.

A method for the determination of testosterone in human plasma is described. Testosterone is separated by thin-layer chromatography, and determined quantitatively by the fluorescence reaction on a surface of lithium hydroxide. As little as $0.01 \mu \mathrm{g}$ testosterone can be determined by the above method.

Kürzlich berichteten wir über eine Methode zur Bestimmung von Testosteron im Harn des Menschen, die darauf beruht, daß Testosteron auf der Oberfläche eines
Lithiumhydroxid-Preßlings eine Fluoreszenz gibt (1). $\mathrm{Da}$ diese Fluoreszenzreaktion spezifisch für alle $\Delta^{4}-3$ Ketosteroide ist, mußte das Testosteron vorher von 


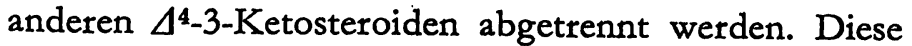
Fluoreszenzreaktion ist sehr empfindlich; noch $0,05 \mu \mathrm{g}$ Testosteron lassen sich in einer Probe bestimmen. Deshalb schien es aussichtsreich, dieses Prinzip auch zur Bestimmung des Testosterons im Plasma zu verwenden.

Zur Bestimmung des Testosterons im Plasma wurden bisher folgende Prinzipien verwendet: Die Doppelisotopentechnik $(2,3,4)$, die Gaschromatographie $(5,6)$ und die enzymatische Uberführung des Testosterons in Östradiol mit anschließender Mikrofluorometrie $(7,8)$. Hierbei ist jedoch der Zusatz von isotopenmarkiertem Testosteron als innerem Wiederfindungsstandard erforderlich. Horton und Mitarbeiter (9) benutzten bei ihrer Methode ein spezifisches testosteronbindendes Protein.

Die meisten der beschriebenen Methoden erfordern zur Reinigung des Testosterons bzw. seiner Derivate mehrere chromatographische Reinigungsschritte und sind deshalb für die routinemäßige Bestimmung weniger geeignet. Bei der von uns beschriebenen Methode gestaltet sich die Isolierung des Testosterons viel einfacher. Nachdem der Plasmaextrakt durch Aluminiumoxid filtriert und von Lipoiden befreit wurde, wird das Testosteron von anderen störenden Steroiden durch nur eine eindimensionale Dünnschichtchromatographie getrennt. Infolgedessen sind die Verluste bedeutend geringer als bei anderen Verfahren, und auf den Zusatz von isotopenmarkiertem Testosteron zur inneren Wiederfindungskontrolle kann verzichtet werden.

Das Plasma des Menschen enthält nur sehr geringe Mengen Testosteron (nach den Angaben in der Literatur durchschnittlich $0,7 \mu \mathrm{g} / 100 \mathrm{~m} l$ bei Männern und $0,1 \mu \mathrm{g} / 100 \mathrm{~m} l$ bei Frauen). Eine Methode, die für praktische Belange geeignet sein soll, muß so empfindlich sein, daß sich eine Testosteron-Bestimmung noch in einem kleinen Plasmavolumen ausführen läßt. Bei der Bestimmung des Testosterons im Harn (1) erzielten wir eine Empfindlichkeit von 0,05 $\mu \mathrm{g}$ Testosteron in einer Probe. Diese Empfindlichkeit schien uns für eine Bestimmung des Testosterons im Plasma nicht ausreichend. Deshalb wurde der Fluoreszenzzusatz des Photometers „Eppendorf“" umgebaut, wodurch sich die Empfindlichkeit aưf das Fünffache steigern ließ. Damit sind wir jetzt in der Lage, Mengen bis zu 0,01 $\mu \mathrm{g}$ Testosteron in einer Probe zu bestimmen. In der Empfindlichkeit reicht unsere Methode an die Doppelisotopenmethoden heran.

\section{Methodik}

Reagenzien

Diätbylätber DAB 6 (Fa. Riedel-de Haën) wird über Natriumdraht destilliert. Er wird in einer braunen Flasche aufbewahrt und soll nicht älter als 3 Tage sein.

Atbylacetat für Chromatographie (Fa. Riedel-de Haën)

$n$-Hexan, tein (Fa. Riedel-de Haën)

Chloroform p. a. (Fa. Riedel-de Haën)

Aluminiumoxid, neutral, Akt. II (Fa. Gebr. Giulini, Ludwigshafen)
Aluminiumoxid $G F_{254}$ für die Dünnschicht-Chromatographie (Fa. E. Merck, Darmstadt)

Metbylenchlorid: 2 l Methylenchlorid werden mit $200 \mathrm{~m} /$ 0,1proz. Kaliumpermanganat-Lösung, mit $200 \mathrm{ml} \mathrm{N} \mathrm{NaOH}$ und dreimal mit je $400 \mathrm{ml}$ Wasser ausgeschüttelt. Nach 24stdg. Trocknen über wasserfreiem Calciumchlorid wird das Lösungsmittel destilliert.

Benzol p. a. (Fa. E. Merck, Darmstadt)

Litbiumbydroxid etwa 98\% LiOH (Fa. E. Merck, Darmstadt) Testosteron (Fa. E. Merck, Darmstadt)

Methanol p. a. (Fa. Riedel-de Haën) wird über eine Füllkörperkolonne destilliert.

\section{Extraktion}

Das Blut wird mit einer vorher mit Heparin ausgespülten Spritze abgenommen und zur Gewinnung des Plasmas sofort zentrifugiert. $5 \mathrm{~m} l$ Plasma von Männern werden dreimal mit je $10 \mathrm{~m} l$ Åthylacetat/Äther $(1: 1 \mathrm{~V} / \mathrm{V})$ extrahiert. Die vereinigten Extrakte werden mit $5 \mathrm{ml} \mathrm{N} \mathrm{NaOH}$ und zweimal mit je $5 \mathrm{~m} l$ dest. Wasser ausgeschüttelt, über wasserfreiem Natriumsulfat getrocknet und im Vakuum am Rotationsverdampfer eingedampft. Den Rückstand bringt man sofort mit dreimal $3 \mathrm{~m} /$ Chloroform auf eine Säule aus neutralem Aluminiumoxid (Akt. II) $(1 \times 5 \mathrm{~cm})$ und wäscht mit $13 \mathrm{~m} /$ Chloroform nach. Das Eluat wird im Vakuum eingedampft. Den Rückstand löst man in $7 \mathrm{~m} /$ Methanol und fügt dann $3 \mathrm{ml}$ dest. Wasser $z u$, worauf die Lipoide ausfallen. Nach Abkühlen überführt man die Lösung in einen Schütteltrichter, spült mit $5 \mathrm{ml} 70$ proz. Methanol nach und extrahiert die Lipoide mit $5 \mathrm{ml}$ n-Hexan. Die wäßrig-methanolische Phase wird im Vakuum am Rotationsverdampfer zur Trockne eingedampft. Den Rückstand überführt man mit Chloroform quantitativ in ein spitzes Zentrifugenröhrchen und dampft die Lösung im Vakuum ein.

\section{Dünnschichtchromatographie}

Der Plasmaextrakt wird in der gleichen Weise wie bei der Bestimmung des Testosterons im Harn (1) dünnschichtchromatographisch auf Aluminiumoxid $\mathrm{GF}_{254}$ im Laufmittelsystem Benzol/ Methylenchlorid/Äthylacetat $(3: 1: 1 \mathrm{~V} / \mathrm{V})$ getrennt. Das Areal der Platte, das das Testosteron enthält, wird abgekratzt. Das Aluminiumoxid eluiert man mit zweimal je $2 \mathrm{ml}$ Methanol.

\section{Quantitative Bestimmung}

Die Methodik der Fluoreszenzmessung von Testosteron auf Lithiumhydroxid-Preßlingen haben wir in unserer früheren Arbeit (1) beschrieben. Wir benutzen ein Photometer „Eppendorf" mit Fluoreszenzzusatz. In den Küvettenhalter wurde an Stelle einer Küvette ein gleich großes Metallklötzchen eingesetzt, das an seiner Vorderseite eine Halterung für den Preßling besaß. Der von der Lichtquelle kommende Strahl traf nach Passieren des Primärfilters ( $\mathrm{Hg} 313+366 \mathrm{~nm}$ für Fluorometrie) auf die Vertiefung in der Mitte des Preßlings. Das von dem Preßling emittierte Fluoreszenzlicht gelangte durch ein Filter (530 bis $3000 \mathrm{~nm}$ ) auf die Photozelle. Bei dieser Anordnung saß der Preßling jedoch zu hoch, so daß nur ein Teil des Fluoreszenzlichtes zur Photozelle gelangte. Immerhin konnte man mit dieser Anordnung Mengen bis zu 0,05 $\mu \mathrm{g}$ Testosteron quantitativ bestimmen.

Zur Bestimmung noch kleinerer Mengen Testosteron haben wir diese Anorordnung verändert (Abb. 1). Der von der Lichtquelle $L$ kommende Strahl gelangt durch das Filter $\mathrm{Hg} 313+366 \mathrm{~nm}$ und trifft auf einen kleinen Spiegel S, wo er nach unten abgelenkt wird. Der Preßling P sitzt jetzt tiefer und befindet sich in der Nähe des Filters F (530-3000 nm). Das von dem Preßling emittierte Fluoreszenzlicht tritt durch dieses Filter und trifft auf die dahinter befindliche Photozelle. Auf diese Weise gelangt mehr Fluoreszenzlicht auf die Photozelle. Die Empfindlichkeit konnte durch diese Veränderung um das Fünffache erhöht werden. Es ist jetzt möglich, noch 0,01 $\mu \mathrm{g}$ Testosteron in einer Probe sicher zu bestimmen. Infolge dieser höheren Empfindlichkeit wurden als Standard nur $0,06 \mu \mathrm{g}$ Testosteron auf einen Preßling aufgetragen. 


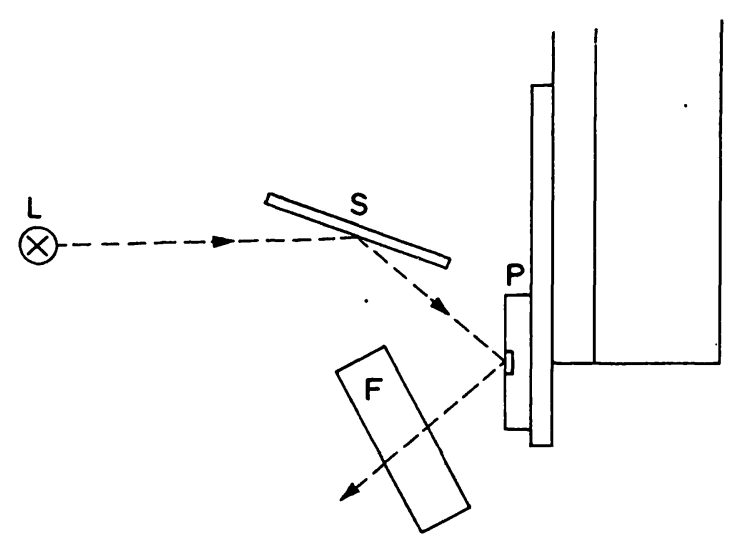

Abb. 1

Anordnung zur Messung der Fluoreszenz auf einem LithiumhydroxidPreßling $\mathrm{L}=$ Lichtquelle, $\mathrm{S}=$ Spiegel, $\mathrm{P}=\mathrm{PiOH}-\mathrm{PreBling}$ mit Testosteron,

\section{Ergebnisse}

Spezifität der Methode

Bei der Methode zur Bestimmung des Testosterons im Harn (1) haben wir ausgeführt, daß nur $\Delta^{4}-3$-Ketosteroide die Fluoreszenzreaktion auf LithiumhydroxidPreßlingen geben. Es wurde gezeigt, daß durch die Dünnschichtchromatographie das Testosteron von anderen $\Delta^{4}-3$-Ketosteroiden getrennt wird. Also ist die Methode spezifisch für Testosteron.

\section{Richtigkeit der Methode}

Zur Bestimmung der Wiederfindung wurden einem Sammelplasma von Frauen, das $0,09 \mu \mathrm{g}$ Testosteron/ $100 \mathrm{ml}$ enthielt, verschiedene Mengen Testosteron zugesetzt. Bei einem Zusatz von 0,04 $\mu \mathrm{g}$ Testosteron pro $5 \mathrm{ml}$ Plasma wurden $75 \%$ und bei einem Zusatz von $0,025 \mu \mathrm{g}$ pro $5 \mathrm{ml}$ Plasma $80 \%$ des zugesetzten Testosterons wiedergefunden (Tab. 1).

Tab. 1

Ergebnisse von Wiederfindungsversuchen, bei denen jeweils $5 \mathrm{~m} l$ Plasma verschiedene Mengen Testosteron zugesetzt wurden

\begin{tabular}{|c|c|c|}
\hline \multicolumn{3}{|c|}{ Testosteron } \\
\hline & $\begin{array}{c}\text { zugesetzt } \\
\mu \mathrm{g}\end{array}$ & $\begin{array}{c}\text { wiedergef. } \\
\mu \mathrm{g}\end{array}$ \\
\hline \multirow{2}{*}{\multicolumn{2}{|c|}{$\begin{array}{l}0,04 \\
0,04 \\
0,04 \\
0,04 \\
0,04 \\
0,04\end{array}$}} & $\begin{array}{l}0,031 \\
0,028 \\
0,031 \\
0,031 \\
0,028 \\
0,032\end{array}$ \\
\hline & & $0,030=75 \%$ \\
\hline " & \multirow[t]{2}{*}{$\begin{array}{l}0,025 \\
0,025 \\
0,025 \\
0,025 \\
0,025 \\
0,025\end{array}$} & $\begin{array}{l}0,023 \\
0,018 \\
0,022 \\
0,016 \\
0,019 \\
0,022\end{array}$ \\
\hline & & $\overline{0,020}=80 \%$ \\
\hline
\end{tabular}

\section{Genauigkeit der Methode}

Der Testosterongehalt eines Sammelplasmas wurde $10 \mathrm{mal}$ bestimmt. Dabei wurden als Mittel der $10 \mathrm{Be}-$ stimmungen $0,41 \mu \mathrm{g} / 100 \mathrm{~m} l$ gefunden. Die Standardabweichung betrug $\mathrm{s}= \pm 0,05 \mu \mathrm{g}( \pm 12 \%$ ) (Tab. 2).
Tab. 2

Genauigkeit der Methode Mehrfachbestimmung von Sammelplasma

\begin{tabular}{c}
$\begin{array}{c}\text { Testosteron im Plasma } \\
\mu \mathrm{g} / 100 \mathrm{ml}\end{array}$ \\
\hline 0,33 \\
0,40 \\
0,37 \\
0,42 \\
0,43 \\
0,41 \\
0,50 \\
0,40 \\
0,37 \\
0,49 \\
\hline $\bar{x}= \pm 0,41$ \\
$\mathrm{~s}= \pm 0,05$ \\
\hline
\end{tabular}

\section{Normalwerte}

Es wurde der Testosterongehalt des Plasmas von 7 Männern im Alter von 22 bis 36 Jahren bestimmt. Die gefundenen Werte sind in Tabelle 3 zusammengefaßt.

Tab. 3

Testosteron im Plasma von Männern

\begin{tabular}{cc}
\hline $\begin{array}{c}\text { Alter } \\
\text { (Jahre) }\end{array}$ & $\begin{array}{c}\text { Testosteron } \\
\mu \mathrm{g} / 100 \mathrm{~m} \bar{l}\end{array}$ \\
\hline 36 & 0,47 \\
22 & 1,01 \\
24 & 0,70 \\
29 & 1,15 \\
30 & 0,99 \\
22 & 0,44 \\
28 & 1,18 \\
\hline
\end{tabular}

\section{Diskussion}

Mit der von uns beschriebenen Methode läßt $\cdot$ sich Testosteron aus einem Plasmaextrakt nach Filtrieren durch eine kleine Aluminiumoxid-Säule sowie Entfernung der Lipoide durch Verteilung zwischen 70proz. Methanol und $\mathrm{n}$-Hexan durch einmalige eindimensionale Dünnschichtchromatographie isolieren. Hierbei wird

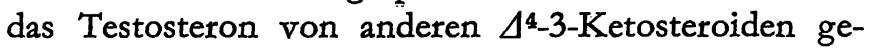
trennt, und nur diese geben eine Fluoreszenz auf der Oberfläche eines Lithiumhydroxid-Preßlings. Die Vorteile dieser wenigen Reinigungsschritte sind: Die Verluste bei der Aufarbeitung sind relativ gering. Auf den Zusatz von isotopenmarkiertem Testosteron als innerem Standard kann daher verzichtet werden. Die Bestimmung läßt sich schnell ausführen. Eine Arbeitskraft kann an einem Tage 3 Bestimmungen ausführen.

Die Methode ist mit der neuen Meßanordnung sehr empfindlich. Man kann noch $0,01 \mu \mathrm{g}$ Testosteron in einer Probe bestimmen. Für eine Bestimmung von Testosteron im Plasma von Männern sind $5 \mathrm{~m} l$ ausreichend. Bei Frauen werden $20 \mathrm{ml}$ Plasma benötigt. Der apparative Aufwand ist gering. Man braucht keinen Gaschromatographen und keine Meßgeräte zur Bestimmung der Radioaktivität.

Die von uns ermittelten Normalwerte von Männern sind mit den Befunden anderer Autoren vergleichbar, sie liegen jedoch höher als die von Oerter (10) sowie von FINKELstein und Mitarbeitern (7) gefundenen Werte. Im Sammelplasma von Frauen fanden wir $0,09 \mu \mathrm{g} / 100 \mathrm{~m} l$. Dieser Wert deckt sich mit den Angaben in der Literatur.

Herrn Albert Brüstle sei für ausgezeichnete Mitarbeit gedankt. 


\section{Literatur}

1. Graef, V., P. Jobst und HJ. Staudinger, diese Z. 6,159 (1968). - 2. Hudson, B., J. Coghlan, A. Dulmanis, M. Wintour und I. Ekker, Austral. J. Exper. Biol. 41, 235 (1963). - 3. RionDEL, A., J. F. TAIt, M. Gut, S. A. S. Tait, E. Joachim und B. LitrLe, J. Clin. Endocr. Metab., Springfield 23, 620 (1963). 4. Burger, H. G., J. R. Kent und A. E. Kelite, J. Clin. Endocr. Metab., Springfield 24, 432 (1964). - 5. BrownIE, A. C., H. J. van der Molen, E. E. Nishizawa und K. B. Erk-Nes, J. Clin. Endocr. Metab., Springfield 24, 1091 (1964). - 6. NAKAGAwA, K.,
N. McNiven, E. Forchielli, A. Vermeulen und R. I. DorfMAN, Steroids 7, 329 (1966). - 7. Finkelstern, M., E. Forchielli und R. I. Dorfman, J. Clin. Endocr. Metab., Springfield 21, 98 (1961). - 8. Forchielli, E., G. Sorcini, M. S. Nightingale, N. Brust, R. I. Dorfman, W. H. Perloff und G. Jacobson, Analyt. Biochem. (New York) 5, 416 (1963). - 9. Horton, R., T. Kato und R. Sherins, Stetoids 10, 245 (1967). - 10. Oertel, G. W., Acta endocr. K'hvn 37, 237 (1961).

Prof. Dr. Hj. Staudinger $63 \mathrm{Gießen}$

Friedrichstr. 24

\section{Lactat- und Pyruvatkonzentrationen im Blut während der Acidose des Neugeborenen}

Von D. KaISER und E. WERner

Aus der Kinderklinik des Städt. Rudolf-Virchow-Krankenbauses Berlin (Ärztl. Direktor: Prof. Dr. E. Werner)

(Eingegangen am 2. April 1968)

Herrn Prof. Dr. Dr. Ernst Schütte qum 60. Geburtstag geividmet

An 18 rcifen Neugeborenen wurde festgestellt, daß die 48 Stdn. dauernde metabolische Acidose korreliert ist mit den Blutlactatwerten. Außerdem fand sich - trotz ausreichenden $\mathrm{O}_{2}$-Angebotes - ein erhöhter Reduktionszustand der Gewebe in diesem Lebensabschnitt. Es konnte gezeigt werden, daß die Bestimmung von Lactat und Pyruvat auch eine klinisch-diagnostische Aussagekraft für die postnatale Periods: besitzt.

A study of 18 mature, newly born infants showed that the metabolic acidosis, which lasts for 48 hrs., is correlated with the concentration of lactate in the blood. Furthermore, there was an increase in the reduction state of the tissue during this period despite a sufficient supply of oxygen. It was shown that the determination of lactate and pyruvate can also be used for diagnostic purposes during the postnatal period.

Störungen des Säure-Basen-Gleichgewichts in der perinatalen Periode sind für den Geburtshelfer und den Pädiater von großem Interesse, weil nach neueren Anschauungen (1) die Acidose weitgehend bestimmt: 1. Den Zeitpunkt aktiven geburtshilflichen Eingreifens 2. Art und Ausmaß der post partum einzuleitenden Maßnahmen der Acidosebekämpfung und damit 3. die Überlebenschancen des Neugeborenen.

Beim reifen Neugeborenen ist die respiratorische Komponente der Acidose schon 60 bis 120 Min. nach der Abnabelung ausgeglichen, wie aus den arteriellen $\mathrm{P}_{\mathrm{CO}_{2}}$-Werten (30-40 Torr) $\mathrm{zu}$ ersehen ist (2).

Unabhängig hiervon verläuft die metabolische Acidose. Viele Untersuchungen der letzten Jahre zeigten, daß sie mit einem Anstieg der Blutlactatkonzentrationen einhergeht. Insbesondere der Zusammenhang zwischen $\operatorname{der} \mathrm{O}_{2}$-Versorgung einerseits, sowie den Lactatkonzen-' trationen und der metabolischen Acidose andererseits, schien uns von Interesse für die Postnatalperiode. Durch gleichzeitige Bestimmung des Säure-Basen-Status ( $\mathrm{pH}$, Standardbicarbonat, $\mathrm{P}_{\mathrm{co}_{2}}$ ), des $\mathrm{P}_{\mathrm{o}_{2}}$, sowie der Lactat- $\left(C_{I}\right)$ und Pyruvatkonzentrationen $\left(C_{P}\right)$ sollte festgestellt werden, wie beim reifen Neugeborenen die metabolische Acidose verläuft, welche Beziehungen zum Lactat bestehen und wie der Reduktionszustand der Gewebe - gemessen am Lactat-Pyruvatquotienten (L/P) $(3,4,5)$ - vom Blutsauerstoff-Angebot abhängt.
Wir fanden, daß die neonatale Acidose vorwiegend in einer Lactatanbäufung bestebt. Sie bält bis 48 Stdn. nach der Geburt an. Trotz ausreichenden $\mathrm{O}_{2}$-Angebotes ist gleichzeitig auch der Redultionszustand der Gevebe (L/P-Quotient) erböbt. Wir balten es für wabrscheinlich, daß dies zurückzufübren ist auf eine geringere Oxydationskapazität des Neugeborenen für Milchsäure.

Nach unserer Meinung stellt die Bestimmung von Lactat und Pyruvat eine wertvolle Bereicherung der Diagnostik in der Postnatalperiode dar.

\section{Methodik}

Die Untersuchungen wurden an 18 reifen, komplikationslos geborenen Säuglingen mit einem Durchschnittsgewicht von $3200 \mathrm{~g}$ über die ersten 48 Lebensstunden durchgeführt. Zur Standardisierung der Stoffwechselbedingungen wurden die Säuglinge eine Stunde vor den Messungen in einen $32^{\circ}$ warmen, d. h. indifferent temperierten Inkubator gelegt $(6,7)$. Aus der hyperaemisierten (Rubriment) und silikonisierten (Hemolube-Salbe) Ferse wurde Kapillarblut in heparinisierte Glasröhrchen entnommen. Zwei Röhrchen wurden zu Bestimmungen von $\mathrm{pH}$, Standardbicarbonat und $\mathrm{P}_{\mathrm{CO}_{2}}$ sowie des $\mathrm{P}_{\mathrm{O}_{2}}$ am Mikro-Kombi-Analysator (Fa. Eschweiler) verwendet.

Zur Lactat- und Pyruvatmessung wurden die ursprünglichen Methoden $(8,9)$ auf Halbmiktoverfahren adaptiert (EPPENDORF Mikrolitersystem) und wie folgt modifiziert: Aus dem Einstich perlendes Fersenblut wird lückenlos in zwei heparinisierte „EscHwEILER-Kapillaren" (etwa $140 \mu l$ ) aufgesaugt, sofort vollständig in eisgekühlte $0,6 \mathrm{~N} \mathrm{HClO}_{4}$ ausgeblasen, gut durchmischt und 60 Sek. bei $18000 \mathrm{~g}$ zentrifugiert. $200 \mu /$ des Überstandes werden mit 100 bis 